\title{
A SUFFICIENT CONDITION FOR NONABELIANNESS OF FUNDAMENTAL GROUPS OF DIFFERENTIABLE MANIFOLDS ${ }^{1}$
}

\author{
KUO-TSAI CHEN
}

Abstract. In this paper we prove that, if $H^{r}(X)$ denotes the $r$ th deRham cohomology group of a connected manifold $X$ and if the cup product $H^{1}(X) \wedge_{R} H^{1}(X) \rightarrow H^{2}(X)$ is not injective, then $\pi_{1}(X)$ is not abelian. As a corollary, if $b_{r}$ is the $r$ th Betti number, then $\frac{1}{2} b_{1}\left(b_{1}-1\right)>b_{2}$ implies $\pi_{1}(X)$ being nonabelian.

Let $X$ be a connected differentiable manifold with a base point $x_{0}$. Denote by $H(X)$ its deRham cohomology over the real (or complex) number field $K$. The purpose of this note is to provide a simple direct proof of the next assertion, which seems to have escaped notice.

THEOREM. If the fundamental group $\pi_{1}(X)$ is abelian, then the canonical homomorphism $\theta$ given by the composition

$$
H^{1}(X) \wedge_{K} H^{1}(X) \rightarrow H^{1}(X) \wedge H^{1}(X) \subseteq H^{2}(X)
$$

is injective, where $H^{1}(X) \wedge_{K} H^{1}(X)$ is the exterior product over $K$ and $H^{1}(X) \wedge H^{1}(X)$ is the cup product. ${ }^{2}$

An immediate consequence is the following sufficient condition for $\pi_{1}(X)$ to be nonabelian.

Corollary. Let $b_{r}=\operatorname{dim}_{K} H^{r}(X)$. If $\frac{1}{2} b_{1}\left(b_{1}-1\right)>b_{2}$, then $\pi_{1}(X)$ is nonabelian.

REMARK. Manifolds having an abelian fundamental group have attracted very early attention. $K$. Reidemeister's work [3] implies that, if a compact 3-manifold $X$ has an abelian fundamental group, then $b_{1} \leqq 3$, which was also proved by P. A. Smith [4]. Now the same in-

Received by the editors November 27, 1969.

AMS 1969 subject classifications. Primary 5731.

Key words and phrases. Fundamental groups, de Rham cohomology groups, iterated path integrals.

1 Work supported in part by the National Foundation under grant NSF GP-13145.

2 It was observed by W. S. Massey that the theorem is valid for CW complexes with finitely generated homotopy groups and for an arbitrary field as cohomology coefficient. His proof consists of a reduction to the case of $K(\pi, 1)$ by attaching $n$-cells, $n \geqq 3$, in order to kill higher homotopy groups. In doing so there is an injection on the level of second cohomology groups. Therefore one has only to verify the theorem for $K(\pi, 1)$ with $\pi$ being an abelian group. 
equality also follows from the above corollary by noting that $b_{2}=b_{1}$.

Proof of TheOREM. Let $\tilde{X}$ be the universal covering space of $X$ with a base point $\tilde{x}_{0}$. If $w$ is a closed 1 -form on $X$, the path integral $\int_{x_{0}} w$ lifts to a function $f_{w}$ on $\tilde{X}$ with $f_{w}\left(\tilde{x}_{0}\right)=0$.

Let $w_{1}, \cdots, w_{m}$ be closed 1-forms, whose cohomology classes $\left[w_{1}\right], \cdots,\left[w_{m}\right]$ are linearly independent. If

$$
u=\sum_{i<j} c_{i j}\left[w_{i}\right] \wedge\left[w_{j}\right] \in \operatorname{Ker} \theta,
$$

then there exists a 1 -form $w$ with $d w=\sum_{i<j} c_{i j} w_{i} \wedge w_{j}$. Let $\alpha:[0,1] \rightarrow X$ be a (piecewise smooth) path from $x_{0}$, which lifts to a path $\tilde{\alpha}$ from $\tilde{x}_{0}$ in $\tilde{X}$. Define

$$
\int_{\alpha} w_{i} w_{j}=\int_{0}^{1}\left(\int_{\alpha \mid[0, t]} w_{i}\right) w_{j}(\alpha(t), \dot{\alpha}(t)) d t .
$$

Then

$$
\int_{\tilde{\alpha}} \sum c_{i j} w_{i} w_{j}=\int_{\alpha} \sum c_{i j} f_{w_{i}} \tilde{w}_{j}
$$

The exterior derivative of the 1 -form $\sum c_{i j} f_{w_{i}} \tilde{w}_{j}$ on $\tilde{X}$ is simply $\sum c_{i j} \tilde{w}_{i} \wedge \tilde{w}_{j}=d \tilde{w}$.

It follows that $\sum c_{i j} f_{w_{i}} \tilde{w}_{j}-\tilde{w}$ is a closed 1 -form on $\tilde{X}$ and that the value of the integral

$$
\int \sum c_{i j} w_{i} w_{j}-\int w
$$

along a loop $\alpha$ at $x_{0}$ depends only on the homotopy class $\langle\alpha\rangle \in$ $\pi_{1}\left(X, x_{0}\right)$.

Choose loops $\alpha_{1}, \cdots, \alpha_{m}$ at $x_{0}$ such that the $m \times m$ matrix $\left(\int_{\alpha_{j}} w_{i}\right)$ is nonsingular. We may assume that $\int_{\alpha_{j}} w_{i}=\delta_{i j}$. (Normalize the $w$ 's if necessary.) Write $\alpha_{i j}=\alpha_{i} \alpha_{j} \alpha_{i}^{-1} \alpha_{j}^{-1}$. Then $\int_{\alpha_{i j}} w=0$. Use the formulas

$$
\int_{\alpha \beta} w w^{\prime}=\int_{\alpha} w w^{\prime}+\int_{\alpha} w \int_{\beta} w^{\prime}+\int_{\beta} w w^{\prime}
$$

and

$$
\int_{\alpha \alpha^{-1}} w w^{\prime}=0
$$

to verify that 


$$
\begin{aligned}
\int_{\alpha \beta \alpha^{-1} \beta-1} w w^{\prime}= & \int_{\alpha \beta} w w^{\prime}+\int_{\alpha \beta} w \int_{\alpha^{-1} \beta-1} w w^{\prime}+\int_{\alpha^{-1} \beta-1} w w^{\prime} \\
= & \int_{\alpha} w w^{\prime}+\int_{\alpha} w \int_{\beta} w+\int_{\beta} w w^{\prime} \\
& -\left(\int_{\alpha} w+\int_{\beta} w\right)\left(\int_{\alpha} w^{\prime}+\int_{\beta} w^{\prime}\right) \\
& +\int_{\alpha^{-1}} w w^{\prime}+\int_{\alpha} w \int_{\beta} w^{\prime}+\int_{\beta^{-1}} w w^{\prime} \\
= & \int_{\alpha} w \int_{\beta} w^{\prime}-\int_{\beta} w \int_{\alpha} w^{\prime} .
\end{aligned}
$$

If $c_{l k} \neq 0, l<k$, then the value of the integral (1) along $\alpha_{l k}$ is nonzero, and $\alpha_{l k}$ cannot be nullhomotopic. Since $\pi_{1}(X)$ is abelian, this cannot happen, and $u=0$. Every element of $\operatorname{Ker} \theta$ can be written in the form of $u$. Hence $\theta$ is injective.

The above result and further extensions can be also taken as applications of a systematic treatment of iterated path integrals, which will be presented in a forthcoming work [2]. The algebraic aspect of the work has been announced in [1].

\section{REFERENCES}

1. K. T. Chen, An algebraic dualization of fundamental groups, Bull. Amer. Math. Soc. 75 (1969), 1020-1024.

2. - Algebras of iterated path integrals, (to appear).

3. P. A. Smith, Manifolds with abelian fundamental groups, Ann. of Math (2) 37 (1936), 526-533.

4. K. Reidemeister, Kommutative Fundamentalgruppen, Monatsh. Math. Phys. 43(1936), 20-28.

UNIVERSITY OF ILLINOIS, URBANA, ILLINOIS 61801 